DYNAMICAL SYSTEMS AND ERGODIC THEORY BANACH CENTER PUBLICATIONS, VOLUME 23 PWN - POLISH SCIENTIFIC PUBLISHERS

WARSZAWA 1989

\title{
HOW COMPLICATED CAN BE ONE DIMENSIONAL DYNAMICAL SYSTEMS: DESCRIPTIVE ESTIMATES OF SETS
}

\author{
A. N. SHARKOVSKII \\ Institute of Mathematics, Academy of Sciences of the Ukrainian S.S.R. \\ Kiev, U.S.S.R.
}

In the theory of dynamical systems, along with open or closed sets such as basins of sinks, nonwandering sets, centers of dynamical systems, one considers sets with more complicated structure. There appear $F_{\sigma}$ sets, which are unions of not more than countably many closed sets, such as the set of all periodic points, $G_{\delta}$ sets, which are intersections of not more than countably many open sets, such as the set of all orbitally stable points, $F_{\sigma \delta}$ sets, which are intersections of not more than countably many $F_{\sigma}$ sets etc. Sometimes instead of the above Hausdorff classification one uses Baire (Lusin-de la Vallée Poussin) classification. In the latter, open sets and closed sets together with all sets which are both $F_{\sigma}$ and $G_{\delta}$ belong to the first class. The second class consists of sets which are $F_{\sigma}$ sets or $G_{\delta}$ but not both and of sets which are at the same time $F_{\sigma \delta}$ and $G_{\delta \sigma}$ but do not belong to the first class. Further classes are defined in a similar way.

Usually upper descriptive estimates are obtained easily for dynamical systems on an arbitrary space with countable basis of its topology. It turns out that those upper estimates can be reached already on one dimensional systems. Therefore, from the point of view of descriptive set theory, one dimensional dynamical systems can be as complicated as dynamical systems on arbitrary topological spaces ${ }^{(1)}$. Later on we shall give descriptive estimates for the sets most frequently used in the theory of dynamical systems, which are related to such notions as attraction of points, their stability, recurrence.

1. Let $(X, f)$ be a dynamical system with discrete time, let $X$ be a separable

(1) In some sense this statement is no surprise: the real line is so rich that it meets almost all needs of descriptive set theory. On the other hand, if we restrict our attention to those dynamical systems which are group actions (as opposed to semigroups) then the dynamics on the real line is quite simple and it suffices to deal with open or closed sets only. 
metric space and $f$ a continuous map $X \rightarrow X$. We shall study mainly one dimensional dynamical systems for which $X=I$ is an interval on the real line.

The orbit through a point $x \in X$, i.e. $\bigcup_{i \geqslant 0}\left\{f^{i}(x)\right\}$, is an at most countable subset of $X$. It is always of type $F_{\sigma}$, this statement holds also for dynamical systems with continuous time although in that case the orbit is in general uncountable. Already in the case of $X=I$, as it is well known, there can exist orbits dense in some perfect set, e.g. for the map $x \rightarrow 4 x(1-x)$ of the interval $I=[0,1]$ almost all orbits are dense in $I$ and therefore they are not $G_{\delta}$ sets.

The precise statement is as follows. Let $\operatorname{Per}(f)$ be the set of all periodic points. For a map $f \in C^{0}(I, I)$ there exists an orbit which is not $G_{\delta}$ set iff Per $f$ is not a closed set [1]. If $f$ possesses a periodic point of a period $\neq 2^{i}, i$ $=0,1,2, \ldots$ then $\operatorname{Per}(f)$ is not a closed set and moreover it is not a $G_{\delta}$ set [1].

This means in particular that the situation in which the upper estimate (valid for an arbitrary dynamical system) is reached on an interval, is fairly typical, so it makes sense to expect the same for other sets considered in the theory of dynamical systems.

\section{Returning points}

In the theory of dynamical systems one considers different types of return of sets and points. If return means that open sets or at least their parts return at least once, like in the case of nonwandering points, then points with such a property form a closed set. If we have in mind a stronger type of return, eg. periodicity, almost periodicity, recurrence, Poisson stability, then in general we shall obtain more complicated sets.

Recall the definitions. A point $x \in X$ is almost periodic if for every neighborhood $U$ of $x$ it is possible to find $m>0$ such that $f^{m i}(x) \in U$ for $i$ $=1,2, \ldots$

If for every $i$ there is $l, 0 \leqslant l<m$, such that $f^{m i+l}(x) \in U$, then $x$ is recurrent. If for an arbitrary neighborhood $U$ of a point $x$ one can find $m>0$ such that $f^{m}(x) \in U$, then $x$ is Poisson stable ${ }^{(2)}$.

If the notion of an $\omega$-limit set of a point $x, \omega_{f}(x)=\bigcap_{n>0} \bigcup_{i \geqslant n}\left\{f^{i}(x)\right\}$, is under consideration, then the last two definitions may be restated as follows: $x$ is Poisson stable if $x \in \omega_{f}(x)$; if in addition $\omega_{f}(x)$ is a minimal set (i.e. it does not contain proper closed invariant subsets) then $x$ is called recurrent.

\footnotetext{
(2) The notion of Poisson stability is widely used in Russian language literature. In English the word "recurrence" is used in this case. Points which are recurrent according to our definition are referred to as almost periodic.
} 
Denote by $\operatorname{Ap}(f)$ the set of all almost periodic points, by $\operatorname{Rec}(f)$ the set of all reccurent points and. by Pois $(f)$ the set of all Poisson stable points.

The set $\operatorname{Per}(f)$ is always of type $F_{\sigma}$ because it is the union of countably many closed sets Fix $\left(f^{m}\right)=\left\{x \in X: f^{m}(x)=x\right\}$. The set Pois $(f)$ is always a $G_{\delta}$ set - the proof of this statement is almost the same as the proof of the analogous theorem for groups of maps in [2]. Both descriptive estimates are reached in the one dimensional case. If a map $f \in C^{0}(I, I)$ has a periodic point of prime period $\neq 2^{i}, i=0,1,2, \ldots$ then both $\operatorname{Per}(f)$ and $\operatorname{Pois}(f)$ belong to the second Baire class, i.e. $\operatorname{Per}(f)$ is not a $G_{\delta}$ set while $\operatorname{Pois}(f)$ is not an $F_{\sigma}$ set. The last statement follows from the fact that if $f$ has a periodic point of prime period $\neq 2^{i}$ then there exists an invariant closed set $F$ in which points which are not Poisson stable are everywhere dense and there exists an everywhere dense orbit [3]. Since Pois $(f) \cap F$ is a $G_{\delta}$ set dense in $F$, the set $F \cap(I \backslash$ Pois $(f))$ also dense in $F$ cannot be of type $G_{\delta}$, notice that two $G_{\delta}$ sets dense in $F$ always do intersect. Therefore Pois $(f)$ cannot be an $F_{\sigma}$ set.

It is plausible that descriptive estimates for $\operatorname{Rec}(f)$ and $\operatorname{Ap}(f)$ for general dynamical systems, can also be reached in the one dimensional case. Up to now this remains a conjecture. We can prove only two theorems

TheOREM 1. If $f \in C^{0}(X, X)$, then $\operatorname{Rec}(f)$ and $\operatorname{Ap}(f)$ are $F_{\sigma \delta}$ sets.

THEOREM 2. If $f \in C^{0}(I, I)$ has a periodic point of prime period $\neq 2^{i} i$ $=0,1,2, \ldots$, then $\operatorname{Rec}(f)$ is not $a G_{\delta}$ set and $\operatorname{Ap}(f)$ is neither an $F_{\sigma}$ nor $G_{\delta}$ set.

We start with the proof of Theorem 2. If $f$ has a periodic point of prime period $\neq 2^{i}$ then there exists a closed invariant set $F$ with a dense orbit and with periodic points dense in $F$ [3]. In this case the sets $\operatorname{Rec}(f)$ and $\operatorname{Ap}(f)$ are dense in $F$, because they contain $\operatorname{Per}(f)$. Also, the set $P=\left\{x \in F: \omega_{f}(x)\right.$ $=F\}$ is dense in $F$. Since $P$ is a $G_{\delta}$ set [4], neither $\operatorname{Rec}(f)$ nor $\operatorname{Ap}(f)$ can be $G_{\delta}$ sets as they do not intersect $P$ (points in $P$ are not recurrent).

$\operatorname{Ap}(f)$ is not an $F_{\sigma}$ set because there exist minimal sets in which all points except a countable many are almost periodic. In such a situation $\operatorname{Ap}(f)$ is not an $F_{\sigma}$ set and since the minimal sets are closed, $\operatorname{Ap}(f)$ is not $F_{\sigma}$ as a subset of $I$.

Let us go back to Theorem 1. Let $\Sigma=\left\{\sigma_{j}^{s}: s=1,2, \underset{s_{j}}{,}, s_{j}, s_{j} \leqslant \infty\right.$, $j=1,2, \ldots\}$ be an open basis of $X$, let for each $j=1,2, \ldots \bigcup_{s=1} \sigma_{j}^{s}=X$, and let for arbitrary $x \in X$ and its neighborhood $U$ there is $j$ such that if $j>j^{\prime}$ then $\sigma_{j}^{s} \subset U$, provided $x \in \sigma_{j}^{s}$.

We are going to show that

$$
\operatorname{Ap}(f)=\left\{x \in X: \forall U \ni x \exists m>0, f^{m \cdot i}(x) \in U \text { for } i>0\right\}
$$


is always an $F_{\sigma \delta}$ set.

For every $\sigma \in \Sigma$ and every $m>0$ we define $A_{m}(\sigma)=\bigcap_{i=1}^{\infty}\left(\bar{\sigma} \cap f^{-m \cdot i}(\bar{\sigma})\right)$. For every $\sigma \in \Sigma$ we define an $F_{\sigma}$ set $A_{*}(\sigma)=\bigcup_{m=1}^{\infty} A_{m}(\sigma)$ and for every $j>0$ an $F_{\sigma}$ set $A^{j}=\bigcup_{s=1}^{s_{j}} A_{*}\left(\sigma_{j}^{s}\right)$. The claim is that the $F_{\sigma \delta}$ set $A=\bigcap_{j=1}^{\infty} A_{j}$ equals $\operatorname{Ap}(f)$, i.e.

$$
\operatorname{Ap}(f)=\bigcap_{j=1}^{\infty} \bigcup_{s=1}^{s_{j}} \bigcup_{m=1}^{\infty} \bigcap_{i \geqslant 1}\left(\bar{\sigma}_{j}^{s} \cap f^{-m \cdot i}\left(\bar{\sigma}_{j}^{s}\right)\right) .
$$

In fact, if $x \in \operatorname{Ap}(f)$ and $\{x\}=\bigcap_{j=1}^{\infty} \sigma_{j}^{r_{j}}$ then for every $j \geqslant 1$ there is $m_{j}>0$ such that $f^{m_{j} \cdot i}(x) \in \sigma_{j}^{r_{j}}, i>0$. Therefore $x \in A_{m_{j}}\left(\sigma_{j}^{r_{j}}\right)$ for $j=1,2, \ldots$ and thus $x \in A^{j}=\bigcup_{s=1}^{s_{j}} \bigcup_{m=1}^{\infty} A_{m}\left(\sigma_{j}^{s}\right)$ and $x \in A=\bigcap_{j} A^{j}$ i.e. $\operatorname{Ap}(f) \subset A$.

On the contrary, if $x \in A$ then $x \in A^{j}$ for every $j \geqslant 1$, so for every $j$ there is $r_{j}, 1 \leqslant r_{j} \leqslant s_{j}, m_{j}>0$ such that $x \in A_{m_{j}}\left(\sigma_{j}^{r_{j}}\right)$. This means that $x \in \operatorname{Ap}(f)$, since $\sigma_{j}^{r_{j}}$ are sequeezed to the point $x$ as $j \rightarrow \infty$. Hence $A \subset \operatorname{Ap}(f)$.

In an analogous way it can be shown that $\operatorname{Rec}(f)=\{x \in X: \forall U \ni x$ $\exists m>0, U \cap \bigcup_{l=1}^{m}\left\{f^{-m \cdot i+l}(x)\right\} \neq \emptyset$ for every $\left.i>0\right\}$ is an $F_{\sigma \delta}$ set, and moreover

$$
\operatorname{Rec}(f)=\bigcap_{j=1}^{\infty} \bigcup_{s=1}^{s_{j}} \bigcup_{m=1}^{\infty} \bigcap_{j \geqslant 1}\left(\overline{\sigma_{j}^{s}} \cap \bigcup_{l=1}^{m} f^{-m \cdot i+l}\left(\overline{\sigma_{j}^{s}}\right)\right) .
$$

The problem of the exactness of the estimates in Theorem 1 remains open also in the case of an arbitrary topological space $X$, although it does not look too complicated.

\section{Attracted points}

Let $F$ be an arbitrary closed invariant set. We shall discuss a problem of complexity of the set which consists of all points attracted by $F$ ("stable manifold"), i.e. $P(F)=\left\{x \in X: \omega_{f}(x)=F\right\}$. If $F$ is an attractor which does not contain nontrivial closed invariant subsets, then $P(F)$ is an open set. In this case the following more precise result has been obtained [4]:

the set $\left\{x \in X: \omega_{f}(x) \supset F\right\}$ is always of type $G_{\delta}$;

the set $\left\{x \in X: \omega_{f}(x) \subset F\right\}$ is always of type $F_{\sigma \delta}$

and therefore $P(F)$ is of type $F_{\sigma \delta}$, too. 
All these upper bounds can be reached already in the one dimensional case even in the case of $F$ being one point set, i.e. when $F$ is a fixed point [5]. For example, the set $P(\{0\})$ for the map

$$
x \stackrel{f_{0}}{\rightarrow} \begin{cases}x+x \sin 1 / x & \text { if } x \neq 0, \\ 0 & \text { if } x=0\end{cases}
$$

is not of the type $G_{\delta \sigma}$. The map may look somewhat strange but if $F$ is not a cycle then on "most of occasions" $P(F)$ will be precisely of that type and not of the type $G_{\delta \sigma}$ : if and only if in any neighborhood of the $\omega$-limit set $F$ there is another $\omega$-limit set $F^{\prime} \supset F, P(F)$ is not a $G_{\delta \sigma}$ set, so it is of the third Baire class [6]. Such $\omega$-limit sets exist iff $f$ has a cycle of prime period $\neq 2^{i}$, in which case most of the sets are of this sort. $[3,6]^{(3)}$.

Do sets of class $>3$ show up among sets which appear in a natural way in the theory of dynamical systems? It seems that unless some more precise classifications of points are considered we shall meet only sets of class $\leqslant 3$ (and in general, sets of class $\leqslant r+2$, provided $f$ is of Baire class $r$ ). Nonetheless, there may appear more complicated sets, too. If we are interested not only in the set $P(F)$ but in its subset $P^{*}(F)$ characterized by some special type of convergence of its points toward $F$, then $P^{*}(F)$ may turn out to be of class $>3$.

The simplest way of giving an idea of what may happen is to look at well known arithmetic examples of sets of classes $\geqslant 3$. The Baire example of a set of class 3 is an $F_{\sigma \delta}$ set $B \subset[0,1]$ built up of points whose continued fraction expansion $\left[n_{1}, n_{2}, \ldots\right]$ has the property that $n_{i} \rightarrow \infty$ as $i \rightarrow \infty$. L. Keldysh example of set of class 4 is subset $B^{*}=\left\{x \in B: \forall s \geqslant 0 \exists N_{s}, 2^{s}\right.$ is a divisor of card $\left\{i: n_{i}=n\right\}$ for $\left.n>N_{s}\right\}$. For the discontinuous map

$$
x \rightarrow \begin{cases}\{1 / x\} & \text { if } x \neq 0 \\ 0 & \text { if } x=0\end{cases}
$$

$P\left(\left\{0_{1}^{\prime}\right)=B\right.$ up to the $F_{\sigma}$ set of all rational numbers, the set $B^{*}$ is made up of points of $P(\{0\})$ such that for any $s>0$ the number of its visits to all intervals of type $\left(\frac{1}{n+1}, \frac{1}{n}\right)$ is a multiple of $2^{\text {s }}$ begining from some number $n$ that depends on $s$.

(3) Later, analogous theorems have been proved also for expanding maps on manifolds Sharkovskit AN., Bondarchuk V. S., Partially ordered system of $\omega$-limit sets of expanding maps, in "Dynamical systems and stability problems for solutions of differential equations", (in Russian), Institute of Mathematics of Ukrainian Academy of Sciences, Kiev 1973, 128-164). For wider classes of dynamical systems including smooth maps on manifolds, the problems of this sort have been almost out of consideration. 


\section{Homoclinic points}

There is another set, which may have a complicated structure, namely the set of homoclinic points [7]. Denote it by $H(f)=\left\{x \in X: \omega_{f}(x)\right.$ is a cycle and there exists a sequence $x_{-1}, x_{-2}, \ldots, f\left(x_{-i}\right)=x_{-i+1}, x_{0}=x$, attracted to $\left.\omega_{f}(x)\right\}$. The set $H(f)$ is always of type $F_{\sigma \delta \sigma}$.

It is unknown if this estimate can be reached for one dimensional systems or even for more general systems. For the map $f_{0}$ defined in section 3. $H\left(f_{0}\right)$ is in the third class for it is $F_{\sigma \delta}$ but not $G_{\delta \sigma}[7,8]$. The set $H(f)$ may belong to the fourth class only in the case of a map $f$ which has a countable number of cycles being attracting and repelling simultaneously such as the point 0 in the case of $f_{0}$. If the map does not have such cycles then the structure of the set $H(f)$ is much simpler, in general $H(f)$ is an $F_{\sigma}$ set. If $f \in C^{0}(I, I)$, then $H(f) \neq \emptyset$ iff $f$ has a cycle of prime period $\neq 2^{i}$, in this case $H(f)$ is not a $G_{\delta}$ set.

\section{Stable orbits}

In the theory of dynamical systems one considers different types of stability such as Liapunov stability, asymptotic stability, orbital stability, stability under permanent perturbation. Stability of the orbit through a point $x$ usually is equivalent to continuity at $x$ of some map, which corresponds to the type of stability under consideration. Since the set of continuity of a map is always a $G_{\delta}$ set, points lying on stable orbits usually form a $G_{\delta}$ set in $X$.

What maps correspond to one or another type of stability? In the case of Liapunov stability the corresponding map is $X \rightarrow X^{\omega}, x \rightarrow(\dot{x}, f(x)$, $\left.f^{2}(x), \ldots\right)$, in the case of the orbital stability $-X \rightarrow 2^{X}\left(x \rightarrow \operatorname{pr}_{f}(x)=\right.$ $\left.\bigcap_{U x i=0}^{\infty} f^{i}(U)\right)$, for orbital stability under permanent perturbation $-X$
$\times C^{0}(X, X) \rightarrow 2^{X}$,

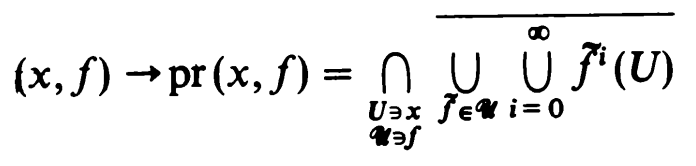

and so on. It is not difficult to understand that upper descriptive estimates for the sets consisting of points of stable orbits can be reached in the one dimensional case.

It is not less important to classify dynamical systems themselves than to classify points, e.g. those, which are "globaly" stable in one sense or another. We are not going into the details of the problem of descriptive estimates of sets of maps, say in $C^{0}(X, X)$, which have these or other dynamical properties, but it is worth to mention that such estimates (as descriptive estimates for stable orbits) may be quite useful for proving that some other types of orbits or dynamical systems are generic [9]. 


\section{References}

[1] A. N. Sharkovskii, On cycles and structure of a continuous map, Ukrain. Math. Zh. 17 (3) (1965), 104-111 (in Russian).

[2] V. V. Nemycki, V. V. Stepanov, Qualitative theory of differential equations, Moskva, OGIZ, 1949 (in Russian).

[3] A. N. Sharkovskii, Partially ordered system of attracting sets, Dokl. Akad. Nauk SSSR 170 (6) (1966) 1276-1278 (in Russian).

[4] -, On attracting and attracted sets, Dokl. Akad. Nauk. SSSR 160 (5) (1965), 1036-1038 (in Russian) (English translation: Soviet 'Math. Dokl. 6 (1965)).

[5] -, On a classification of fixed points, Ukrain. Math. Zh. 17 (5) (1965); 80-95, (in Russian) (English translation: Amer. Math. Soc. Transl. 97 (2) (1970), 159-179.

[6] -, The behaviour of a map in a neighbourhood of an attracting set, Ukrain. Math. Zh. 18 (2) (1966), 60-83 (in Russian) (English translation: Amer. Math. Soc. Transl. 97 (2) (1970), 227-258).

[7] -, Descriptive estimates of the set of homoclinic points of a dynamical system, in Differentialdifference equations and mathematical physics problems, edited by Math. Inst. of Ukrainian Acad. of Sciences, Kiev 1984, 109-115 (in Russian).

[8] -, On the set of convergence of one dimensional iterations, Dopovidi Akad. Nauk Ukrain RSR 7 (1966), 866-870 (in Ukrainian).

[9] V. A. Dobrynskii, A. N. Sharkovskii, Genericity of dynamical systems, almost all orbits of which are stable under permanently scting perturbations, Dokl. Akad. Nauk SSSR 211 (2) (1973) 273-276, (in Russian) (English translation: Soviet Math. Dokl. 14 (4) (1973)). 